\title{
Prevalence of Clonorchis sinensis infection in dogs and cats in subtropical southern China
}

\author{
Rui-Qing Lin ${ }^{1,2}$, Jian-Dong Tang ${ }^{1,2}$, Dong-Hui Zhou' ${ }^{1}$, Hui-Qun Song ${ }^{1}$, Si-Yang Huang ${ }^{1}$, Jia-Xu Chen ${ }^{3}$, Mu-Xin Chen³, \\ Han Zhang ${ }^{4}$, Xing-Quan Zhu ${ }^{1,5,6^{*}}$ and Xiao-Nong Zhou ${ }^{3^{*}}$
}

\begin{abstract}
Background: Clonorchiasis, caused by Clonorchis sinensis, is one of the major parasitic zoonoses in China, particularly in China's southern Guangdong province where the prevalence of $C$. sinensis infection in humans is high. However, little is known of the prevalence of $C$. sinensis infection in its reservoir hosts dogs and cats. Hence, the prevalence of $C$. sinensis infection in dogs and cats was investigated in Guangdong province, China between October 2006 and March 2008.

Results: A total of 503 dogs and 194 cats from 13 administrative regions in Guangdong province were examined by post-mortem examination. The worms were examined, counted, and identified to species according to existing keys and descriptions. The average prevalences of C. sinensis infection in dogs and cats were $20.5 \%$ and $41.8 \%$, respectively. The infection intensities in dogs were usually light, but in cats the infection intensities were more serious. The prevalences were higher in some of the cities located in the Pearl River Delta region which is the most important endemic area in Guangdong province, but the prevalences were relatively lower in seaside cities.

Conclusions: The present investigation revealed a high prevalence of $C$. sinensis infection in its reservoir hosts dogs and cats in China's subtropical Guangdong province, which provides relevant "base-line" data for conducting control strategies and measures against clonorchiasis in this region.
\end{abstract}

\section{Background}

Clonorchiasis, caused by Clonorchis sinensis, is considered as one of the major parasitic zoonoses in some parts of Asia, which can cause a series of hepatic diseases such as periductal inflammation, fibrosis, pyogenic cholangitis, biliary calculi, cholecystitis, liver cirrhosis and pancreatitis, and C. sinensis is currently classified as carcinogenic to humans for cholangiocarcinoma [1-3]. It is estimated that about 35 million people are infected globally, and of whom approximately 15 million are in China $[4,5]$, where human clonorchiasis is endemic in 27 provinces (including municipal cities and autonomous regions) [4-6].

\footnotetext{
* Correspondence: xingquanzhu1@hotmail.com; ipdzhouxn@sh163.net ${ }^{1}$ State Key Laboratory of Veterinary Etiological Biology, Key Laboratory of Veterinary Parasitology of Gansu Province, Lanzhou Veterinary Research Institute, Chinese Academy of Agricultural Sciences, Lanzhou, Gansu Province 730046, PR China

${ }^{3}$ National Institute of Parasitic Diseases, Chinese Center for Disease Control and Prevention, Shanghai 200025, PR China

Full list of author information is available at the end of the article
}

Snails serve as the first intermediate hosts for $C$. sinensis and the prevalence is high (up to 15\%) in some areas of China, and approximately 140 species of freshwater fishes and four species of shrimp have been recognized as the second intermediate hosts for $C$. sinensis in China [6]. Many mammals, such as cats, dogs and pigs, serve as definitive hosts or reservoir hosts for $C$. sinensis $[4,7,8]$. "Ingesting raw fish" is considered to be the main risk factor for human infection with $C$. sinensis $[4,9,10]$. Dogs and cats are the most important animal reservoirs for human infection with $C$. sinensis. There are about two hundred million dogs and one hundred million cats in China [11]. Since the 1990s, epidemiological studies of $C$. sinensis infection in dogs and cats have been conducted in many provinces, such as Guizhou, Sichuan, Guangxi, Heilongjiang, Jiangsu, and Hunan [12-18], and the prevalences are summarized in Table 1, because these data were published in local Chinese journals which are not readily accessible to international readers. In general, the prevalence of $C$. sinensis infection in dogs and cats is high but it differs in different areas.
C Biomed Central

C 2011 Lin et al; licensee BioMed Central Ltd. This is an Open Access article distributed under the terms of the Creative Commons Attribution License (http://creativecommons.org/licenses/by/2.0), which permits unrestricted use, distribution, and reproduction in any medium, provided the original work is properly cited. 
Table 1 Prevalence of Clonorchis sinensis infection in dogs and cats in other provinces/cities of the People's Republic of China

\begin{tabular}{|c|c|c|c|c|c|c|}
\hline Species & Province/city & No. tested & Positive (\%) & Test method $^{a}$ & Year tested & References \\
\hline \multirow[t]{6}{*}{ Dog } & Hunan & 438 & 29.4 & PE & $2006-2007$ & Dai et al. (2009) [18] \\
\hline & Heilongjiang & 178 & 26.4 & PE & $1996-2004$ & Wang et al. (2006) [16] \\
\hline & Guangxi & 110 & 56.4 & $P E$ and $F E$ & 1990-1997 & Li et al. (2002) [15] \\
\hline & Sichuan & 891 & 25.6 & $\mathrm{FE}$ & 1978-1985 & Qu et al. (1997) [13] \\
\hline & Jiangsu/Xinyi & 99 & 48.5 & $P E$ and $F E$ & 1998-2006 & Suo et al. (2008) [17] \\
\hline & Guizhou & 120 & 0.8 & PE & 1991-1993 & Wang et al. (1995) [12] \\
\hline \multirow[t]{5}{*}{ Cat } & Hunan/Changsha & 8 & 62.5 & $P E$ and $F E$ & 2000 & Liu et al. (2000) [14] \\
\hline & Guangxi & 78 & 64.1 & $P E$ and $F E$ & 1990-1997 & Li et al. (2002) [15] \\
\hline & Sichuan & 201 & 42.3 & $\mathrm{FE}$ & 1978-1985 & Qu et al. (1997) [13] \\
\hline & Guizhou & 36 & 0 & PE & 1986 & Wang et al. (1995) [12] \\
\hline & Jiangsu/Xinyi & 151 & 62.9 & $\mathrm{PE}$ and $\mathrm{FE}$ & 1998-2006 & Suo et al. (2008) [17] \\
\hline
\end{tabular}

a PE: post-mortem examination; FE: faecal examination

Compared with that of dogs, the prevalence of C. sinensis in cats is obviously higher, possibly due to their preference of eating fish.

Guangdong province is bounded by the South China Sea and is the southern gateway of China. It has the largest number of people (approximately 5.5 million) infected with $C$. sinensis because of the local custom of eating raw and undercooked freshwater fish [6]. Accompanied by the high speed development of economy and the increasing living standards during the last two decades, more and more dogs and cats are being raised and kept in increasing numbers of families as the favorable companion animals in Guangdong province. As reservoir hosts, dogs and cats play an important role in the transmission of $C$. sinensis to humans. Therefore, $C$. sinensis infection in dogs and cats not only remains a severe health problem for dogs and cats, but also poses public health concerns in China. Some surveys have shown that infection of dogs and cats with $C$. sinensis is common in other provinces of China (Table 1). However, prior to the present comprehensive survey, there had been no detailed data of $C$. sinensis prevalence in dogs and cats in Guangdong province.

In order to provide "base-line" data for the improved prevention and control of $C$. sinensis infection in dogs and cats, and for reducing the risk of human infection, the objective of the present investigation was to estimate the prevalence of $C$. sinensis infection in dogs and cats in different geographical locations in southern China's subtropical Guangdong province by post-mortem examination.

\section{Materials and methods}

The study site

Guangdong province is located in the southern part of mainland China, between north latitudes of $20^{\circ} 19^{\prime}$ to $25^{\circ}$ $31^{\prime}$ and east longitudes of $109^{\circ} 45^{\prime}$ to $117^{\circ} 20^{\prime}$, sharing borders with Hong Kong and Macao Special Administrative Regions of China. This province has a subtropical climate, with an average annual temperature of $19^{\circ} \mathrm{C}$ to $23^{\circ} \mathrm{C}$. The province has an area of approximately 180 thousand square kilometers, and has a population of approximately 95 million. People in Guangdong have the habit of eating slices of raw freshwater fishes. Guangdong province has 13 administrative regions (cities), and dogs were sampled from each of these (Table 2). Cats were sampled from 8 of the 13 administrative regions (cities) (Table 3 ), which represent all the main endemic regions for $C$. sinensis.

\section{Examination of dogs and cats for the presence of $C$. sinensis and data collection}

From October 2006 to March 2008, livers from 503 adult farm dogs and 194 adult farm cats sampled randomly from the selected study regions (cities) of Guangdong province were examined for the presence of $C$. sinensis adults. These animals were treated humanely, according to the Animal Ethics Procedures and Guidelines of the People's Republic of China, and the study was approved by South China Agricultural University. Individual livers of dogs and cats were split into two lobes according to anatomical structure, then the bile ducts were opened longitudinally with a pair of scissors in $0.9 \%$ saline, and washed with the same solution until the supernatant had cleared. The sedimentary contents of bile ducts were then carefully examined with the naked eye. All worms were counted if the total number of worms was less than 100, or a proportion of all collected worms were counted if more than 100 worms were estimated to be present. Specimens were fixed in $75 \%$ ethanol after being cleared and species identification was carried out according to existing descriptions and keys (testes and vitelline glands) [4,19]. The identity of three representative recovered worms was ascertained 
Table 2 Prevalence of Clonorchis sinensis infection in dogs in Guangdong province, China

\begin{tabular}{lllll}
\hline Cities surveyed & No. examined & No. positive & Prevalence (\%) & Infection intensity (worms per dog) \\
\hline Jiangmen & 20 & 3 & 15 & $0(17), 1-10(1), 11-50(1),>100(1)$ \\
Guangzhou & 14 & 1 & 7.1 & $0(13), 1-10(1)$ \\
Shantou & 68 & 1 & 1.5 & $0(67), 1-10(1)$ \\
Yunfu & 36 & 13 & 36.1 & $0(23), 1-10(7), 11-50(4), 51-100(2)$ \\
Maoming & 44 & 0 & 0 & $0(44)$ \\
Shaoguan & 49 & 34 & 69.4 & $0(15), 1-10(12), 11-50(9), 51-100(5),>100(8)$ \\
Foshan & 21 & 4 & 19.0 & $0(17), 1-10(2), 51-100(1),>100(1)$ \\
Zhaoging & 42 & 11.9 & $0(37), 1-10(4),>100(1)$ \\
Meizhou & 48 & 2 & 0 & $0(36), 1-10(3), 11-50(2), 51-100(4),>100(3)$ \\
Qingyuan & 22 & 9 & 9.1 & $0(20), 1-10(2)$ \\
Shenzhen & 23 & 0 & 39.1 & $0(14), 1-10(3), 11-50(1), 51-100(3),>100(2)$ \\
Yangjang & 10 & 19 & 0 & $0(10)$ \\
Zhanjiang & 106 & 103 & 17.9 & $0(400), 1-10(10), 11-50(4),>100(2)$ \\
Total & 503 & 20.5 & $(21), 51-100(15),>100(18)$ \\
\hline
\end{tabular}

by the amplification and subsequent sequence analysis of the internal transcribed spacers (ITS) of nuclear ribosomal DNA following methods reported previously [20].

A dog or cat was recorded as infected if at least one C. sinensis worm was found in that animal. The mean prevalence was calculated by dividing the number of infected animals with the total number of animals examined, and was expressed as a percentage. Infection intensity was divided into 5 grades: $0,1-10,11-50,51-100$, > 100 (worms per dog or cat).

\section{Results}

The worms found in livers of dogs and cats showed classical morphological features of $C$. sinensis. The worms were flat, leaf-like, about 6-17 $\mathrm{mm}$ in length and 1-3 $\mathrm{mm}$ in width, with branched testes in tandem position and the continuously distributed vitelline glands. PCR amplification of ITS rDNA of representative worms and subsequent sequence analysis demonstrated that the examined worms had almost identical ITS sequences to that of $C$. sinensis reported previously [20] (not shown).

Of 503 examined dogs, 103 (20.5\%) were positive for C. sinensis in Guangdong province, and the infection intensity in dogs were mainly light, with $47.6 \%$ of the positive dogs being infected with 1 to 10 adult worms in the liver, but $17.5 \%$ of the positive dogs being infected with more than 100 adult worms (Table 2). Usually, high prevalence was accompanied with high intensity. For example, the prevalence of $C$. sinensis in dogs in Shaoguan was the highest, being $69.4 \%$, with high infection intensity-13 dogs of 34 positive dogs were infected with more than 50 worms.

Of the 194 cats, 81 (41.8\%) were infected with $C$. sinensis (Table 3), and the prevalence in cats in Guangzhou, the capital of Guangdong province, was the highest, being $73.2 \%$. The prevalence of $C$. sinensis in cats in Guangdong province was significantly higher than that in dogs $(P<0.05)$. The infection intensity was also high in cats, and even thousands of worms were found in some cats, obviously due to their eating preference for fish.

\section{Discussion}

It is important to study the transmission and epidemiological features of $C$. sinensis infection in order to effectively control human clonorchiasis. Epidemiological

Table 3 Prevalence of Clonorchis sinensis infection in cats in Guangdong province, China

\begin{tabular}{lllll}
\hline Cities surveyed & No. examined & No. positive & Prevalence (\%) & Infection intensity (worms per cat) \\
\hline Jiangmen & 18 & 12 & 66.7 & $0(6), 1-10(7), 11-50(1), 51-100(2),>100(2)$ \\
Guangzhou & 41 & 30 & 73.2 & $0(11), 1-10(6), 11-50(12), 51-100(3),>100(9)$ \\
Shantou & 47 & 1 & 2.1 & $0(46), 1-10(1)$ \\
Yunfu & 20 & 12 & 60 & $0(8), 1-10(7), 11-50(5)$ \\
Foshan & 26 & 9 & 34.6 & $0(17), 1-10(4), 11-50(3),>100(2)$ \\
Qingyuan & 6 & 2 & 33.3 & $0(4), 1-10(2)$ \\
Yangjiang & 19 & 9 & 47.4 & $0(10), 1-10(2), 11-50(3), 51-100(2),>100(2)$ \\
Zhanjiang & 17 & 6 & 35.3 & $0(11), 1-10(5), 11-50(1)$ \\
Total & 194 & 81 & 41.8 & $0(113), 1-10(34), 11-50(25), 51-100(7),>100(15)$ \\
\hline
\end{tabular}


studies of $C$. sinensis infection in humans and/or in intermediate hosts have been studied in South Korea, Japan, and China $[2,8,10,21,22]$. The prevalence of $C$. sinensis in many animal reservoir hosts such as dogs and cats have also been investigated in Korea and some provinces of China $[7,8,12-18]$, but is very limited in many other Chinese provinces including Guangdong province.

Clonorchiasis is the most serious and important parasitic zoonosis in Guangdong province where approximately 5.5 million people were infected with $C$. sinensis $[4,6,23]$, which accounts for approximately one-third of the total population infected with $C$. sinensis in China $[4,6]$. The present investigation represents the first comprehensive survey of $C$. sinensis infection in its reservoir hosts dogs and cats in Guangdong province. The average prevalence of $C$. sinensis infection in dogs and cats were $20.5 \%$ and $41.8 \%$, respectively, which is a little lower than that in some other surveys in other endemic provinces such as Guangxi [15] (Table 1), but were still unacceptably high. These results demonstrated that $C$. sinensis infection in dogs and cats was common in Guangdong province and would play an important role in the transmission of $C$. sinensis between animals and humans.

The prevalence of $C$. sinensis infection in dogs and cats was different among different geographical localities in Guangdong province. The prevalence was relatively higher in some of the cities located in the Pearl River Delta region, such as $39.1 \%$ in dogs in Shenzhen, $66.7 \%$ and $73.2 \%$ in cats in Jiangmen and Guangzhou, respectively. The Pearl River Delta region is economically the most important area in Guangdong province, and there is a high prevalence of $C$. sinensis infection in freshwater fish and shrimps which are the second intermediate hosts for $C$. sinensis $[4,24]$. In the present study, dogs and cats in some cities which are located out of the Pearl River Delta region were also seriously infected with C. sinensis, for example $69.4 \%$ and $36.1 \%$ in dogs in Shaoguan and Yunfu, and $60 \%$ in cats in Yunfu,

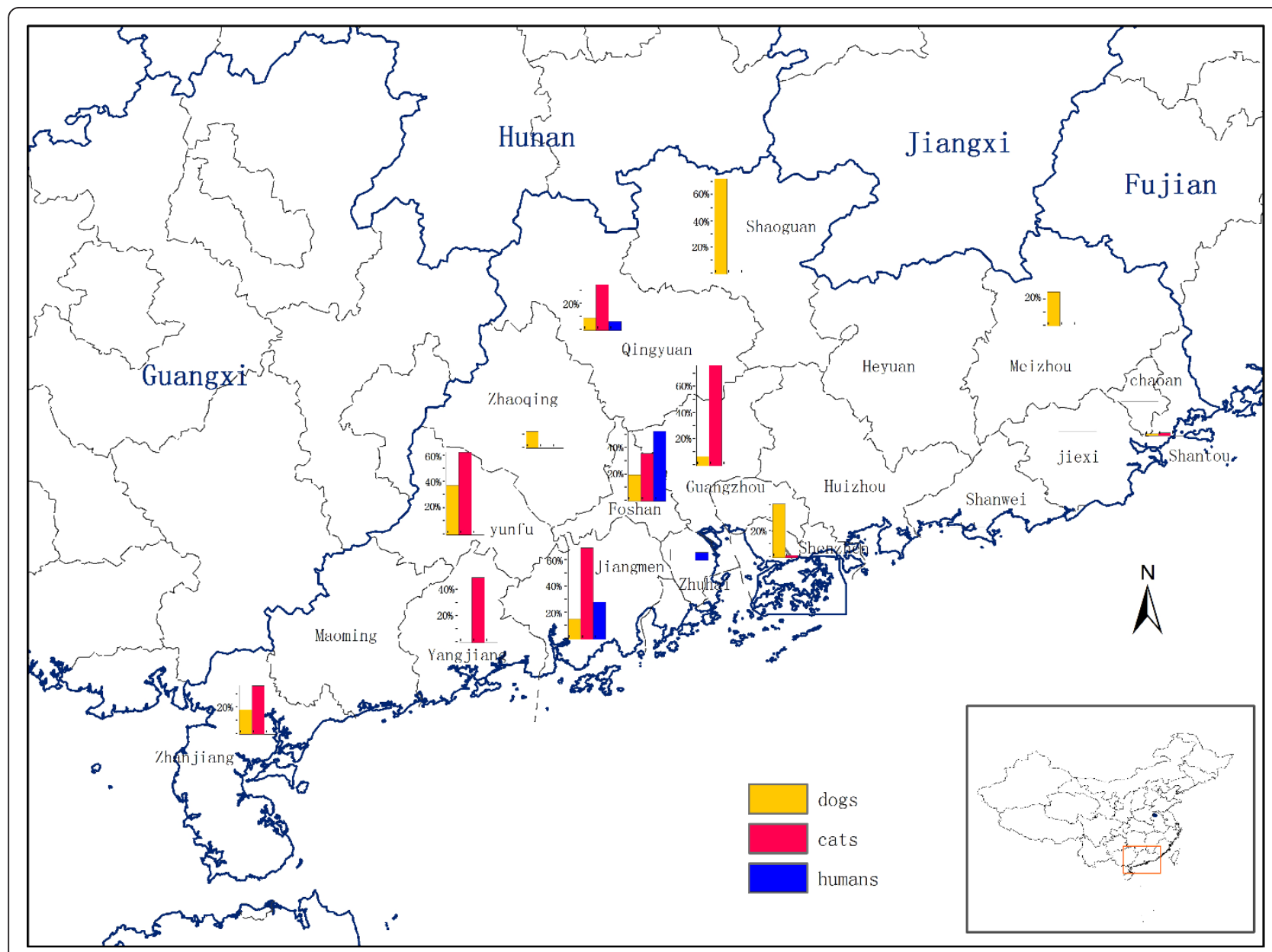

Figure 1 Geographical locations and respective prevalence of Clonorchis sinensis infection in dogs, cats and humans [23]in Guangdong province. 
respectively, indicating that strategies and measures against clonorchiasis should also be used in these regions and cities.

The prevalence of $C$. sinensis in dogs and cats was relatively lower in the seaside cities including Shantou, Zhanjiang, Maoming and Yangjiang (Tables 2 and 3, Figure 1). For example, the prevalence of $C$. sinensis in dogs $(1.5 \%)$ and cats $(2.1 \%)$ in Shantou (the eastern city of Guangdong province) was the lowest, which may due to differences in ecological, geographical and socioeconomic conditions. It is interesting to note that dogs in Shenzhen had a high prevalence of C. sinensis (39.1\%), but the prevalence of $C$. sinensis in humans in this city was very low $(1.6 \%,[23])$. This is probably because Shenzhen is the biggest immigration city in southern China, the majority of its inhabitants were migrants from non-endemic areas, and they do not have the habit of eating raw or undercooked freshwater fish.

\section{Conclusions}

The present survey demonstrated the high prevalence of C. sinensis infection in dogs and cats in southern China's subtropical Guangdong province where human clonorchiasis represents a major health problem, which poses a significant risk for public health. Integrated strategies and measures should be taken to tackle the problem of the high prevalence of $C$. sinensis infection in dogs and cats in this province, which, in turn, would have impact on the effective control and eventual elimination of human clonorchiasis.

\section{Acknowledgements \\ This work was supported, in part, by the National S \& T Major Program (Grant No. 2008ZX10004-011, 2009ZX10004-302, 2009ZX10004-201), the National Key Technology R \& D Program (Grant No. 2008BAI56B03), the State Key Laboratory of Veterinary Etiological Biology, Lanzhou Veterinary Research Institute, Chinese Academy of Agricultural Sciences (Grant Nos. SKLVEB2009KFKT014, SKLVEB2010KFKT010 and SKLVEB2011KFKT004), and the Yunnan Provincial Program for Introducing High-level Scientists (Grant No. 2009(1125).}

\section{Author details}

${ }^{1}$ State Key Laboratory of Veterinary Etiological Biology, Key Laboratory of Veterinary Parasitology of Gansu Province, Lanzhou Veterinary Research Institute, Chinese Academy of Agricultural Sciences, Lanzhou, Gansu Province 730046, PR China. ${ }^{2}$ College of Veterinary Medicine, South China Agricultural University, Guangzhou, Guangdong Province 510642, PR China. ${ }^{3}$ National Institute of Parasitic Diseases, Chinese Center for Disease Control and Prevention, Shanghai 200025, PR China. ${ }^{4}$ Guangdong Institute for Animal Disease Control and Surveillance, Guangzhou, Guangdong Province 510230, PR China. ${ }^{5}$ College of Animal Science and Technology, Yunnan Agricultural University, Kunming, Yunnan Province 650201, PR China. ${ }^{6}$ College of Animal Science and Veterinary Medicine, Heilongjiang Bayi Agricultural University, Daqing, Heilongjiang Province 163319, PR China.

\section{Authors' contributions}

XQZ and XNZ conceived and designed the study, and critically revised the manuscript. SYH, JXC, MXC and HZ participated in study design, study implementation and manuscript revision. RQL, JDT, DHZ and HQS performed the experiments, analysed the data and drafted the manuscript. SYH, JXC,
MXC and $\mathrm{HZ}$ helped in study implementation and data collection. All authors read and approved the final manuscript.

\section{Competing interests}

The authors declare that they have no competing interests.

Received: 12 August 2011 Accepted: 19 September 2011 Published: 19 September 2011

\section{References}

1. Chai JY, Murrell KD, Lymbery AJ: Fish-borne parasitic zoonoses: Status and issues. Int J Parasitol 2005, 35:1233-1254.

2. Shin HR, Oh JK, Lim MK, Shin A, Kong HJ, Jung KW, Won YJ, Park S, Park SJ, Hong ST: Descriptive epidemiology of cholangiocarcinoma and clonorchiasis in Korea. J Korean Med Sci 2010, 25:1011-1016.

3. Sun J, Xu J, Liang P, Mao Q, Huang Y, LV X, Deng C, Liang C, de Hoog GS, Yu X: Molecular identification of Clonorchis sinensis and discrimination with other opisthorchid liver fluke species using multiple Ligationdepended Probe Amplification (MLPA). Parasit Vectors 2011, 4:98.

4. Lun ZR, Gasser RB, Lai DH, Li AX, Zhu XQ, Yu XB, Fang YY: Clonorchiasis: a key foodborne zoonosis in China. Lancet Infect Dis 2005, 5:31-41.

5. Li T, He S, Zhao H, Zhao G, Zhu XQ: Major trends in human parasitic diseases in China. Trends Parasitol 2010, 26:264-270.

6. Zhou P, Chen N, Zhang RL, Lin RQ, Zhu XQ: Food-borne parasitic zoonoses in China: perspective for control. Trends Parasitol 2008, 24:190-196.

7. Kim BJ, Ock MS, Kim IS, Yeo UB: Infection status of Clonorchis sinensis in residents of Hamyang-gun, Gyeongsangnam-do, Korea. Korean J Parasitol 2002, 40:191-193.

8. Rim HJ: Clonorchiasis: an update. J Helminthol 2005, 79:269-281.

9. Yu SH, Kawanaka M, Li XM, Xu LQ, Lan CG, Rui L: Epidemiological investigation on Clonorchis sinensis in human population in an area of south China. Jpn J Infect Dis 2003, 56:168-171.

10. Zhang R, Gao S, Geng Y, Huang D, Yu L, Zhang S, Cheng J, Fu Y: Epidemiological study on Clonorchis sinensis infection in Shenzhen area of Zhujiang delta in China. Parasitol Res 2007, 101:179-183.

11. Wang L, Li K, Xie FQ, J SX: Zoonoses in haman, dog and cat. Chin J Vet Med 2002, 38:43-44, (in Chinese).

12. Wang DD, Liu XM, Han HB: Prevalence of helminthes in dogs and cats in Guizhou Province. Chin J Vet Sci Technol 1995, 25:13-15, (in Chinese).

13. Qu ZQ, Zeng MG, Li JY, Chen RX: Epidemiology of clonorchiasis in sichuan, China. J Pract Parasitic Dis 1997, 5:4-7, (in Chinese).

14. Liu NM, J XD, Zeng SH: Epidemiological investigation of clonorchiasis in Xianwei Lake, Changsha, Hunan province. Pract Prevent Med 2000, 7:375, (in Chinese)

15. Li SL, He G, Wei MB, Tan YG, Zhu QY, Shang SM, Zhang LT, Huang KL, Zhu FZ: Epidemiological investigation of clonorchiasis in Guangxi province. Chin J Parasitic Dis Contr 2002, 15:214-216, (in Chinese).

16. Wang CR, Qiu JH, Zhao JP, Xu LM, Yu WC, Zhu XQ: Prevalence of helminthes in adult dogs in Heilongjiang Province, the People's Republic of China. Parasitol Res 2006, 99:627-630.

17. Suo GH, Zhang ZC, Li HM, Lou PA: Epidemiological investigation on clonorchiasis in Xinyi City. J Pathog Biol 2008, 3:insert 1-2, (in Chinese)

18. Dai RS, Li ZY, Li F, Liu DX, Liu W, Liu GH, He SW, Tan MY, Lin RQ, Liu Y, Zhu $X Q$ : Severe infection of adult dogs with helminths in Hunan Province, China poses significant public health concerns. Vet Parasitol 2009, 160:348-350

19. Kaewkes S: Taxonomy and biology of liver flukes. Acta Trop 2003, 88:177-186.

20. Lee SU, Huh S: Variation of nuclear and mitochondrial DNAs in Korean and Chinese isolates of Clonorchis sinensis. Korean J Parasitol 2004, 42:145-148.

21. Kino H, Inaba H, Van De N, Van Chau L, Son DT, Hao HT, Toan ND, Cong LD, Sano M: Epidemiology of clonorchiasis in Ninh Binh Province, Vietnam. Southeast Asian J Trop Med Public Health 1998, 29:250-254.

22. Ju YH, Oh JK, Kong HJ, Sohn WM, Kim JI, Jung KY, Kim YG, Shin HR: Epidemiologic study of Clonorchis sinensis infestation in a rural area of Kyongsangnam-do, South Korea. J Prev Med Pub Health 2005, 38:425-430.

23. Fang YY, Wu J, Liu Q, Huang SY, Lin RX, Zhang QM, Ruan CW: Investigation and analysis on epidemic status of clonorchiasis in Guangdong province. Chin J Pathog Biol 2007, 2:54-56, (in Chinese). 
24. Chen D, Chen J, Huang J, Chen X, Feng D, Liang B, Che Y, Liu X, Zhu C,

Li X, Shen H: Epidemiological investigation of Clonorchis sinensis infection in freshwater fishes in the Pearl River Delta. Parasitol Res 2010, 107:835-839

doi:10.1186/1756-3305-4-180

Cite this article as: Lin et al:: Prevalence of Clonorchis sinensis infection

in dogs and cats in subtropical southern China. Parasites \& Vectors 2011

4:180.

Submit your next manuscript to BioMed Central and take full advantage of:

- Convenient online submission

- Thorough peer review

- No space constraints or color figure charges

- Immediate publication on acceptance

- Inclusion in PubMed, CAS, Scopus and Google Scholar

- Research which is freely available for redistribution

Submit your manuscript at www.biomedcentral.com/submit 